\title{
A Nordic multicenter survey of long-term bowel function after transanal endorectal pull-through in 200 patients with rectosigmoid Hirschsprung disease
}

\author{
Bjornland, Kristin
}

2017-09

Bjornland , K , Pakarinen, M P , Stenstrom , P , Stensrud , K J , Neuvonen, M , Granström , A L , Graneli , C , Pripp , A H , Arnbjörnsson , E , Emblem , R , Wester , T , Rintala , R J \& Nordic Pediat Surgery Study Consor 2017 , ' A Nordic multicenter survey of long-term bowel function after transanal endorectal pull-through in 200 patients with rectosigmoid Hirschsprung disease ' , Journal of Pediatric Surgery , vol. 52 , no. 9 , pp. 1458-1464 . https://doi.org/10.1016/j.jpeds

http://hdl.handle.net/10138/299387

https://doi.org/10.1016/j.jpedsurg.2017.01.001

publishedVersion

Downloaded from Helda, University of Helsinki institutional repository.

This is an electronic reprint of the original article.

This reprint may differ from the original in pagination and typographic detail.

Please cite the original version. 


\title{
A Nordic multicenter survey of long-term bowel function after transanal endorectal pull-through in 200 patients with rectosigmoid

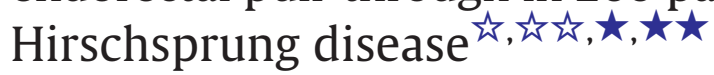

\author{
Kristin Bjørnland ${ }^{\mathrm{a}, *}$, Mikko P. Pakarinen ${ }^{\mathrm{b}}$, Pernilla Stenstrøm ${ }^{\mathrm{c}}$, Kjetil J. Stensrud ${ }^{\mathrm{a}}$, Malla Neuvonen ${ }^{\mathrm{b}}$, \\ Anna L. Granström ${ }^{\text {d }}$, Christina Graneli ${ }^{c}$, Are H. Pripp ${ }^{\text {e }}$, Einar Arnbjörnsson ${ }^{\text {c }}$, Ragnhild Emblem ${ }^{\text {a }}$, \\ Tomas Wester ${ }^{\mathrm{d}}$, Risto J. Rintala ${ }^{\mathrm{b}}$ on behalf of the Nordic Pediatric Surgery Study Consortium \\ a Department of Paediatric Surgery, Oslo University Hospital-Rikshospitalet and University of Oslo, Oslo, Norway \\ ${ }^{\mathrm{b}}$ Department of Paediatric Surgery, Children's Hospital, Helsinki University Hospital and University of Helsinki, Helsinki, Finland \\ c Department of Paediatric Surgery, Skåne University Hospital, Lund, Sweden \\ d Department of Paediatric Surgery, Karolinska University Hospital, Stockholm, Sweden \\ e Department of Paediatric Surgery, Oslo Centre of Biostatistics and Epidemiology, Research Support Services, Oslo University Hospital, Oslo, Norway
}

\section{A R T I C L E I N F O}

\section{Article history:}

Received 4 October 2016

Received in revised form 7 December 2016

Accepted 3 January 2017

\section{Key words:}

Hirschsprung

Long-term bowel function

Pull-through

Fecal incontinence

Enterocolitis

\begin{abstract}
A B S T R A C T
Objective: Transanal endorectal pull-through (ERPT) is the most popular technique to treat Hirschsprung disease (HD). Still, there is limited knowledge on long-term bowel function. This cross-sectional, multicenter study assessed long-term bowel function in a large HD population and examined predictors of poor outcome. Methods: Patients older than four years or their parents filled out a validated questionnaire on bowel function. Clinical details were recorded retrospectively from medical records.

Results: 73/200 (37\%) patients reported absolutely no impaired bowel function, meaning no constipation, fecal accidents, stoma, appendicostomy or need for enemas. Seven (4\%) had a stoma, and 33 (17\%) used antegrade or rectal colonic enemas. Most disarrangements of fecal control and constipation were significantly less common in older age group, but abnormal defecation frequency and social problems remained unchanged. Syndromic patients $(\mathrm{n}=31)$ experienced frequent fecal accidents $(46 \%)$ more often than nonsyndromic $(14 \%, P<0.001)$. Having a syndrome (adjusted OR 5.6, 95\% CI 2.1-15, $P=0.001$ ) or a complete transanal ERPT (adjusted OR 2.4, $95 \% \mathrm{CI} 1.1-5.7, P=0.038$ ) was significantly associated with poor outcome defined as having a stoma, an appendicostomy, daily fecal accidents or need of regular rectal wash outs.

Conclusion: A significant number of HD patients experience bowel problems many years after definite surgery. Fecal control was significantly better in older than younger HD patients, but some continued to have considerable bowel problems also as adults. A total transanal ERPT was associated with poorer outcome. Long-term follow-up of HD patients is warranted.

Prognosis Study: Level II.
\end{abstract}

(c) 2017 Elsevier Inc. All rights reserved.
Hirschsprung disease (HD) is the most common congenital motility disorder in children affecting 1 in 5000 live births [1,2]. Lack of ganglion cells in a variable length of the distal gut causes functional bowel

\footnotetext{
th Funding source: This work was funded by South-Eastern Norway Regional Health Authority.

证败 Financial disclosure: The authors declare no financial relationships relevant to this article.

$\star$ Conflicts of interests: The authors declare no conflicts of interest relevant to this paper.

$\star \star$ Preliminary results were presented at the Annual meetings of the European Association of Paediatric Surgeons, June 2015, Ljubljana, Slovenia and June, 2016, Milan, Italy.

* Corresponding author at: Department of Paediatric Surgery, Oslo University Hospital Rikshospitalet, Postboks 4950, 0424 Oslo. Tel.: +47 230746 20; fax + 723072623.

E-mail address: Kristin.bjornland@medisin.uio.no (K. Bjørnland).
}

obstruction. $80 \%$ of HD patients have aganglionosis restricted to the rectosigmoid colon. There is a strong male preponderance, and other developmental disorders occur in $4 \%-30 \%$ of patients, Down syndrome being the most common [3]. Affected patients usually present shortly after birth with clinical signs of distal intestinal obstruction. In older children, severe constipation is the predominant symptom.

Surgical treatment of HD includes resection of the aganglionic bowel segment and anastomosing ganglionic bowel to the anus. Traditionally, treatment of HD required a colostomy at diagnosis followed by a pullthrough procedure when the child reached 8-12 months. With advances in surgical techniques, neonatal anesthesia and critical care, most children are now operated shortly after diagnosis with a single-stage repair. Different pull-through operations have been described, and minimally invasive techniques have recently become popular. Today the most 
common operations are the Duhamel procedure and the endorectal pull-through, performed either completely transanally (TERPT) or in combination with laparotomy or laparoscopy (LERPT) [4]. TERPT may be considered the least invasive technique because it does not include any transabdominal dissection and leaves no visible scars.

During recent years it has become apparent that long-term morbidity and functional bowel problems after surgical treatment of HD are significant [5,6]. Previous follow-up studies have revealed occurrence of both fecal incontinence and constipation in patients operated with the traditional Duhamel, Soave and Swenson techniques. Obstructive symptoms may be caused by residual aganglionic segment, stricture, or congenital dysmotility, whereas fecal incontinence may be caused of anal sphincter damage, inappropriately low coloanal anastomosis or overflow soiling. So far, there is very limited knowledge concerning the long-term outcome after transanal endorectal pull-through, and in particular, reports where validated questionnaires are used, are few. Most previous studies have included limited number of patients, and duration of follow-up is relatively short. Furthermore, results have mostly been based on retrospective reviews of medical charts. Therefore, we undertook this multicenter study to assess long-term bowel function using validated questionnaires in a large number of HD patients operated with transanal endorectal pull-through with or without transabdominal colonic mobilization. We also analyzed potential predictors of long-term functional outcome.

\section{Methods}

\subsection{Patients}

All HD patients above four years of age with aganglionosis not extending oral to the sigmoid colon operated with primary endorectal pull-through at four Nordic tertiary pediatric surgical centers, were eligible for inclusion. The only exclusion criterion was severe language problems precluding reliable assessment of bowel function.

\subsection{Surgical management}

All patients had the diagnosis verified preoperatively by a rectal biopsy, and a contrast enema, indicating the location of the transition zone, was performed in all patients prior to the pull-through.

The operation was performed either completely transanally (TERPT) or in combination with laparoscopic or laparotomy assisted colonic mobilization (LERPT). A senior consultant either operated or assisted at all operations at all institutions. In some patients a biopsy was taken through a small umbilical incision for exclusion of long-segment HD, and the rest of the operation was performed completely transanally as a TERPT. In patients having a stoma closed simultaneously with the endorectal pull-through, colon was mobilized together with the stoma closure. In all patients a transanal mucosectomy leaving a short muscle cuff was performed. The mucosectomy started approximately $5 \mathrm{~mm}$ above the dentate line and continued for $2-4 \mathrm{~cm}$ [7]. Then, the muscle cuff could be pulled downwards and incised circumferentially. Further dissection of the bowel was continued strictly on the bowel wall. During TERPT, colonic mobilization was performed until the transition zone. If an umbilical biopsy had not been taken at the start of the operation, a frozen section biopsy was obtained to confirm that ganglionic bowel was pulled down to the anal canal. Any dilated bowel above the transition zone was resected together with the aganglionic bowel. For those who underwent LERPT, the transanal mobilization was continued to the level where the colon or rectum had been mobilized from above. A hand-sewn coloanal anastomosis with interrupted sutures was performed in all patients, and no incision in the muscle cuff was performed. The coloanal anastomosis was calibrated in the outpatient clinic during the first postoperative weeks. If the anastomosis felt tight, either the parents continued daily dilatations at home for some weeks, or dilatations were performed regularly in the outpatient clinic.

\subsection{Acquisition of data}

Demographic and clinical details were recorded retrospectively from medical record and included birth weight, associated syndromes and anomalies, family history, presenting symptoms, various surgical details including postoperative complications and reoperations, and occurrence of Hirschsprung associated enterocolitis (HAEC).

Present bowel function was assessed using a bowel function score (BFS) questionnaire developed for assessment of bowel function in pediatric anorectal disorders and validated in patients and in the general population [5,8-10] (Table 1). Soiling refers to fecal staining of underwear, fecal accidents to leakage of fecal contents needing change of underwear or use of protective aids, and constipation was defined as need of a special diet, laxatives or enemas. Patients with a stoma or an appendicostomy for antegrade colonic enemas did not fill out the BFS questionnaire. In addition, there was also a questionnaire for registration of HAEC episodes and milk intolerance. The questionnaires were sent by post and filled out by the patients or the parents, given to the patients or parents at outpatient clinics or filled out by a specialized nurse during a telephone interview. Those who did not return the questionnaires or could not be reached by phone, were sent a reminder. None of the surgeons that had treated the patients were involved in collection of the postoperative functional outcome data.

\subsection{Definitions}

For comparison of results after TERPT and LERPT, those who had an umbilical biopsy, were grouped as having TERPT because no colonic mobilization was done through the umbilical incision. TERPT converted to LERPT were also classified as TERPT. If a stoma was closed simultaneously as the pull-through, the patient was excluded for this particular

Table 1

Bowel function score questionnaire.

\begin{tabular}{|c|c|}
\hline Factor & Score \\
\hline \multicolumn{2}{|l|}{ Ability to hold back defecation } \\
\hline Always & 3 \\
\hline Problems $<1 /$ week & 2 \\
\hline Weekly problems & 1 \\
\hline No voluntary control & 0 \\
\hline \multicolumn{2}{|l|}{ Feels the urge to defecate } \\
\hline Always & 3 \\
\hline Most of the time & 2 \\
\hline Uncertain & 1 \\
\hline Absent & 0 \\
\hline \multicolumn{2}{|l|}{ Frequency of defecation } \\
\hline Every other day to twice a day & 2 \\
\hline More often & 1 \\
\hline Less often & 1 \\
\hline \multicolumn{2}{|l|}{ Soiling } \\
\hline Never & 3 \\
\hline Staining $<1 /$ week, no change of underwear required & 2 \\
\hline Frequent staining, change of underwear often required & 1 \\
\hline Daily soiling, requires protective aids & 0 \\
\hline \multicolumn{2}{|l|}{ Fecal accidents } \\
\hline Never & 3 \\
\hline Fewer $1 /$ week & 2 \\
\hline Weekly, requires protective aids & 1 \\
\hline Daily, requires protective aids day and night & 0 \\
\hline \multicolumn{2}{|l|}{ Constipation } \\
\hline No constipation & 3 \\
\hline Manageable with diet & 2 \\
\hline Manageable with laxatives & 1 \\
\hline Manageable with enemas & 0 \\
\hline \multicolumn{2}{|l|}{ Social problems } \\
\hline No social problems & 3 \\
\hline Sometimes & 2 \\
\hline Problems restricting social life & 1 \\
\hline Severe social/psychosocial problems & 0 \\
\hline
\end{tabular}

The Bowel Function Score questionnaire was used to assess postoperative bowel function in Hirschsprung patients without a stoma or an appendicostomy. 
subanalysis because it was impossible in retrospect to assess the extent of transabdominal colonic mobilization.

The need for anal dilatations for more than three postoperative months was defined as a postoperative stricture. To assess predictors of functional outcome, the patients were divided into two groups based on functional outcome. Poor functional outcome was defined as having a stoma, an appendicostomy, needing regular rectal enemas, or having at least daily fecal accidents as recorded on the BFS questionnaire.

The number of postoperative HAEC episodes was based on review of medical journals and information from patients and parents. When HAEC was recorded from medical records, the diagnosis was based on the presence of abdominal distension, diarrhea, and/or vomiting and/or bloody stools with or without fever.

\subsection{Statistics}

The data are presented as median (range), mean (standard deviation) or frequencies (percentage). IBM SPSS software for Windows version 21.0 (Armonk, NY: IBM Corp.) was used. Categorical variables were compared with a chi-square test. The Gamma coefficient was used as a measure of association of two ordinal variables. Continuous variables were compared between groups with independent samples t-test or Mann-Whitney U-test as appropriate. Predictors for outcome were analyzed using simple and multivariable logistic regression analysis. A $P$ value $<0.05$ was considered statistically significant.

\subsection{Ethics}

The regional ethical review boards at each hospital approved the study.

\section{Results}

\subsection{Patients}

A total of 258 patients with rectosigmoid HD were operated with a primary transanal endorectal pull-through at the four hospitals until 2010. The technique was introduced in the departments in Helsinki, Oslo, Stockholm, and Lund in 1987, 1999, 2001, and 2005, respectively. $55 / 258$ patients/parents did not respond, and three were excluded owing to language problems. Consequently, 200 patients (169 males) were enrolled. 75, 68, 30 and 27 patients were recruited from the hospitals in Helsinki, Oslo, Stockholm, and Lund, respectively. Down syndrome was the most common syndrome (16/31); others were Mowat-Wilson syndrome, congenital central hypoventilation syndrome, Currarino syndrome, hypoplastic left heart syndrome, and others.

A dropout analysis comparing included and nonincluded patients showed that nonincluded patients were older. Median age in included patients was 9.5 years (3.9-32) as compared to 14 years (5.2-31) in those that did not take part in the study $(\mathrm{p}<0.001)$. The frequencies of patients with syndromes (15\% in included patients and $24 \%$ in nonincluded patients; $\mathrm{p}=0.14$ ) and operated with TERPT ( $34 \%$ in included patients and $41 \%$ in nonincluded; $\mathrm{p}=0.1$ ) were similar.

\subsection{Operative details}

Median age at transanal endorectal pull-through was three months (0.4-133). The various operative approaches are outlined in Table 2. Four of the five patients who had TERPT converted to LERPT, were operated in the early period after TERPT introduction, and the last patient had multiple adhesions after a previous laparotomy

\subsection{Surgical complications}

Three patients (1.5\%) developed anastomotic leakage which was treated with a decompressive proximal enterostomy, antibiotics and drainage. One of them also had a fistula to the seminal vesicle. The
Table 2

\begin{tabular}{lllc}
\hline & $\begin{array}{l}\text { Included patients } \\
(\mathrm{n}=200)\end{array}$ & $\begin{array}{l}\text { Not-included patients } \\
(\mathrm{n}=58)\end{array}$ & $P$ \\
\hline Age (years) & $9.5(3.9-32)$ & $14(5.2-31)$ & $<0.001$ \\
Syndrome & $31(15 \%)$ & $4(24 \%)$ & 0.14 \\
TERPT & $67(34 \%)$ & $7(41 \%)$ & 0.17 \\
\hline
\end{tabular}

Operative details for Hirschsprung patients with rectosigmoid aganglionosis. Numbers and frequencies (\%) are given.

enterostomies were closed in all three some months later. Anastomotic stricture occurred in 19 patients (10\%), and all were successfully treated by anal dilatations. A redo endorectal pull-through was performed in three patients (1.5\%) owing to twisted pulled-through colon, fistula to the urethra or transition zone pull-through. The initial operative approaches were LERPT in two and TERPT in one patient. Other recorded complications were adhesive bowel obstruction $(n=4)$, stoma needing revision $(n=2)$, wound infection and/or dehiscence $(n=4)$. In addition, a myectomy or one or more intersphincteric injections of botulinum toxin were performed in five and 22 patients, respectively, to relieve bowel outlet obstruction.

\subsection{Postoperative HAEC}

HAEC was the initial symptom of HD in 14 children (7\%). Information on postoperative HAEC was achieved in 195 patients. Among these, 69 (35\%) reported to have had median 2 (range 1-8) HAEC episodes. The HAEC rate was similar in males (58/153, 38\%) and females $(11 / 42,26 \% ; P=0.159)$. The frequency of HAEC was significantly higher in patients who reported often/always compared to those with rare/ never soiling ( $47 \%$ versus $20 \%, P=0.006$ ). Fecal accidents, constipation or diarrhea was not associated with the number of HAEC episodes (results not shown). Interestingly, patients who got abdominal discomfort of cow's milk had significantly more often experienced HAEC as compared to those who did not get any abdominal symptoms from milk (51\% versus 23\%, $P<0.001$ ). Altogether 73 patients reported some form of discomfort related to milk, but only 22 of these had documented lactose intolerance.

\subsection{Long-term bowel function}

73/200 (37\%) patients reported absolutely no impaired bowel function, meaning that there were no need for bowel management, no constipation, no fecal accidents, and no stoma or appendicostomy. Seven (3.5\%) patients had a stoma, and 33 (17\%) needed bowel management (appendicostomy $(n=16)$ ) or regular rectal wash-outs $(n=17)$.

Only patients $(\mathrm{n}=177$ ) without a colostomy or an appendicostomy filled out the BFS questionnaire. The majority had more than two bowel movements per day (Table 3), and age was not associated with frequency of bowel movements (gamma $=0.18$ ). Constipation was reported in 44 patients (25\%) and was more common in the youngest patients $(P=0.008)$. Also the ability to hold back defecation and recognition of need to defecate were significantly better in the older age groups ( gamma $=0.54$ and 0.35 , respectively).

Soiling and fecal accidents were common and reported in crosssection by $77 \%$ and $47 \%$ of the patients without a stoma or an appendicostomy, respectively (Table 3 ). Both soiling and fecal accidents were less common among the older patients (gamma $=0.44$ and gamma $=0.34$ respectively). Among the teenagers and adults, 93\% experienced no fecal accidents or fecal accidents less than once a week as opposed to 76\% in those below 13 years $(P=0.006)$.

The majority of patients that filled out the BFS questionnaire reported no social problems related to bowel function. Still, bowel problems caused restrictions in social life or severe social/psychosocial difficulties in $11 \%$. Soiling $(P=0.001)$, fecal accidents $(P=0.001)$, impaired 
Table 3

\begin{tabular}{|c|c|c|c|c|c|c|}
\hline Factor & $\begin{array}{l}\text { Overall } \\
(\%)\end{array}$ & $\begin{array}{l}4-7 \text { years } \\
(\%)\end{array}$ & $\begin{array}{l}\text { 8-12 years } \\
(\%)\end{array}$ & $\begin{array}{l}\text { 13-17 years } \\
(\%)\end{array}$ & $\begin{array}{l}>18 \text { years } \\
(\%)\end{array}$ & Gamma/CI/P \\
\hline \multicolumn{7}{|l|}{ Ability to hold back } \\
\hline Always & 51 & 31 & 45 & 78 & 77 & 0.54 \\
\hline Problems $<1$ per wk & 22 & 21 & 30 & 7 & 18 & $(0.4-0.7)$ \\
\hline Problems $>1$ per wk & 24 & 43 & 21 & 3 & 5 & $P<0.001$ \\
\hline No control & 6 & 5 & 4 & 3 & 0 & \\
\hline \multicolumn{7}{|l|}{ Reports urge to defecate } \\
\hline Always & 44 & 32 & 40 & 53 & 76 & 0.35 \\
\hline Mostly & 31 & 30 & 36 & 28 & 24 & $(0.2-0.5)$ \\
\hline Uncertain & 17 & 33 & 9 & 8 & 0 & $P<0.001$ \\
\hline Absent & 8 & 5 & 15 & 11 & 0 & \\
\hline \multicolumn{7}{|l|}{ Frequency of defecation } \\
\hline$<$ every other day & 2 & 6 & 0 & 0 & 0 & 0.18 \\
\hline $2 /$ day to $<$ other day & 45 & 49 & 40 & 40 & 48 & $(0.04-0.4)$ \\
\hline$>2 /$ day & 53 & 45 & 60 & 60 & 52 & $P=0.186$ \\
\hline \multicolumn{7}{|l|}{ Soiling } \\
\hline Never & 23 & 15 & 10 & 42 & 48 & 0.44 \\
\hline$<1 /$ wk & 37 & 27 & 48 & 42 & 39 & $(0.3-0.6)$ \\
\hline$>1 / \mathrm{wk}$ & 27 & 44 & 25 & 11 & 9 & $P<0.001$ \\
\hline Daily & 12 & 15 & 17 & 6 & 4 & \\
\hline \multicolumn{7}{|l|}{ Fecal accidents } \\
\hline Never & 53 & 44 & 45 & 71 & 74 & 0.34 \\
\hline$<1 /$ wk & 28 & 30 & 35 & 20 & 22 & $(0.2-0.5)$ \\
\hline$>1 / \mathrm{wk}$ & 19 & 15 & 8 & 6 & 0 & $P=0.072$ \\
\hline Daily & 9 & 12 & 12 & 3 & 4 & \\
\hline \multicolumn{7}{|l|}{ Constipation } \\
\hline No constipation & 75 & 62 & 78 & 81 & 100 & 0.49 \\
\hline Diet & 3 & 1 & 2 & 8 & 0 & $(0.3-0.7)$ \\
\hline Laxatives & 13 & 20 & 12 & 8 & 0 & $P=0.008$ \\
\hline Enemas & 9 & 16 & 8 & 3 & 0 & \\
\hline \multicolumn{7}{|l|}{ Social problems } \\
\hline No problems & 76 & 72 & 79 & 81 & 74 & 0.08 \\
\hline Sometimes & 13 & 16 & 10 & 11 & 9 & $(-0.2-0.3)$ \\
\hline Restricted social life & 10 & 10 & 8 & 8 & 17 & $P=0.906$ \\
\hline Severe problems & 1 & 2 & 2 & 0 & 0 & \\
\hline Total BFS scores & $16(4-20)$ & $13(5-20)$ & $16(4-20)$ & $18(8-20)$ & $19(13-20)$ & $P<0.001$ \\
\hline
\end{tabular}

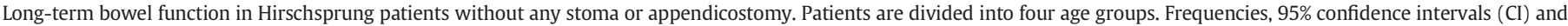
Gamma values are given. For total BFS scores median, minimum and maximum scores are reported.

sensation $(\mathrm{p}=0.001)$, and impaired ability to hold back defecation $(P=$ $0.006)$ were significantly correlated to social problems. In contrast, constipation was not related to social problems $(P=0.7)$.

\subsection{Functional outcome in syndromic patients}

Bowel problems were significantly more common in the syndromic patients (Table 4). The operative approach was similar in syndromic and nonsyndromic patients as TERPT was performed in $46 \%$ and $42 \%$ respectively $(P=0.8)$. As for the total patient population, BFS scores were higher among older than younger syndromic patients $(P=0.04)$.

\subsection{Gender and center related functional outcome}

BFS score was comparable in males and females ( $15.0 \pm 3.8$ versus $15.2 \pm 3.7$, respectively), as was poor outcome (27\% in males, $30 \%$ in females; $P=0.7$ ). $15 \%$ of the males were syndromic and $16 \%$ of the females. Mean age at follow-up was 11.9 and 11.7 years in males and females, respectively. LERPT was performed in $54 \%$ of males and $58 \%$ in females.

The frequency of patients with poor outcome did not differ significantly between the four centers $(23 \%, 23 \%, 31 \%, 37 \% ; P=0.4)$.

\subsection{Predictors of poor functional outcome}

Having a syndrome and undergoing TERPT were significantly associated with poor outcome, whereas age at endorectal pull-through and at follow-up did not influence the frequency of poor outcome in the multivariate model (Table 5).

\section{Discussion}

This multicenter study on a large population of HD patients with rectosigmoid aganglionosis operated with transanal endorectal pull-

Table 4

\begin{tabular}{|c|c|c|c|c|}
\hline & No syndrome $\mathrm{n}=169$ & Syndrome $\mathrm{n}=31$ & & $P$ \\
\hline Age at follow-up (years) & $9.3(3.0-32)$ & $10.6(4-22)$ & & 0.475 \\
\hline Age at operation (months) & $3(0.4-133)$ & $3.6(0.4-59)$ & & 0.904 \\
\hline BFS score & $15.7(15.1-16.2)$ & $10.5(8.6-12.4)$ & MD:5.2 & $<0.001$ \\
\hline Fecal accidents, daily or $>1 /$ week & $14 \%$ & $46 \%$ & OR: $5.3(2.1-13.3)$ & $<0.001$ \\
\hline Constipation Laxatives or enemas & $19 \%$ & $40 \%$ & OR: $2.8(1.2-6.9)$ & 0.023 \\
\hline Stoma or appendicostomy & $10 \%$ & $19 \%$ & OR: $2.1(0.8-5.9)$ & 0.143 \\
\hline
\end{tabular}

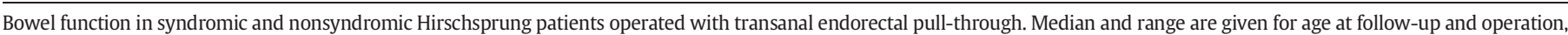
whereas 95\% confidence intervals (CI) are presented for BFS scores and Odds Ratio (OR) values. MD refers to mean difference. 
Table 5

\begin{tabular}{|c|c|c|c|c|c|c|}
\hline Parameter & Poor outcome n (\%) & Not poor outcome n (\%) & Nonadjusted OR (CI) & $P$ & Adjusted OR (CI) & $P$ \\
\hline \multicolumn{7}{|l|}{ Syndrome } \\
\hline Syndrome & $13(57)$ & $10(43)$ & $4(1.9-11.7)$ & 0.001 & $5.64(2.1-15.0)$ & 0.001 \\
\hline No syndrome & $34(21)$ & $123(79)$ & ref & & ref & \\
\hline \multicolumn{7}{|l|}{ Operation } \\
\hline LERPT & $19(20)$ & $78(80)$ & ref & & ref & \\
\hline TERPT & $28(34)$ & $55(66)$ & $2(1.1-4.1)$ & 0.03 & $2.4(1.1-5.7)$ & 0.038 \\
\hline \multicolumn{7}{|l|}{ Age at operation ${ }^{\mathrm{a}}$} \\
\hline Q1 $(0.7 ; 0.4-1)$ & $11(23)$ & $36(77)$ & ref & & ref & \\
\hline Q2 (1.8; 1.0-2.9) & $9(20)$ & $34(79)$ & $0.9(0.3-2.3)$ & 0.8 & $1.4(0.5-4.2)$ & 0.51 \\
\hline Q3 $(4.5 ; 3-7.5)$ & $14(31)$ & $31(69)$ & $1.5(0.6-3.7)$ & 0.4 & $2.3(0.8-6.6)$ & 0.11 \\
\hline Q4 (35.5; 7.9-133) & $13(29)$ & $32(71)$ & $1.2(0.5-3.4)$ & 0.6 & $2.4(0.8-7.0)$ & 0.10 \\
\hline Age at follow-up (years) & $10.0(\mathrm{SD} 4.6)$ & 11.6 (SD6.2) & 0.09 & & $0.97(0.9-1.1)$ & 0.47 \\
\hline
\end{tabular}

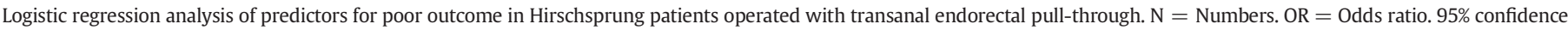
intervals (CI) are given for OR.

${ }^{a}$ Patients are grouped in quartiles (Q) according to age. Mean age in months is given along with minimum and maximum age in each group. Poor outcome was defined as having a stoma, an appendicostomy, daily fecal accidents or use of regular enemas.

through shows that a significant number of patients continue to have long-term problems, mainly related to fecal incontinence. We have also shown that syndromic patients have less favorable functional outcome. Our results also suggest that a completely transanal procedure may predispose to long-term bowel functional defects. Importantly, for the majority of the patients overall bowel function and fecal control seem to improve with increasing age.

Fecal incontinence was the most common problem. Twelve percent of the patients had a stoma or an appendicostomy, mainly because of problems related to fecal incontinence. In addition, $39 \%$ and $18 \%$ of the remaining patients reported soiling or fecal accidents happening more than once a week, respectively. The frequency of fecal incontinence after endorectal pull-through varies considerably in the literature. In a recent retrospective population-based study of 103 patients with rectosigmoid aganglionosis operated with transanal endorectal pullthrough, 29\% reported fecal incontinence episodes of variable degree more than once a week after a median follow-up time of 15 years [11]. High rates of fecal incontinence are also reported in other recent smaller series [6,12]. On the other hand, fecal incontinence was reported in only $12 \%$ in a study of 281 patients operated with endorectal pullthrough after a mean follow-up time of 36 months [13], and in another study of patients undergoing endorectal pull-through, none reported fecal incontinence [14]. There are several plausible explanations for the different outcomes in these studies. First of all, most studies are based on retrospective chart reviews of follow-up visits to the operating surgeon, and not by an independent investigator. There is probably a bias in the surgeon's subjective assessment of postoperative fecal continence. Secondly, in studies assessing outcome after HD surgery, there is no uniform definition of fecal incontinence. When fecal incontinence is examined in adults, it is usual to divide into incontinence for either solid or loose stools, soiling, or urgency, and validated questionnaires are used. In the pediatric literature, several different questionnaires are used, and only the BFS questionnaire has data from the normal population. Furthermore, terms like soiling, staining or fecal accidents have different meanings. In some papers soiling refers to staining of underwear, whereas in other reports soiling includes all grades of fecal incontinence. Thus, a consensus on how to define fecal incontinence after surgical treatment for HD would be helpful. Lastly, HD is a rare disease, and very few hospitals treat a large number of patients. Comparison of outcomes obtained from small patient populations is hampered by the lack of statistical power.

Constipation was reported in $25 \%$ of patients not having a stoma or an ACE. Of these, $9 \%$ needed enemas to manage their constipation and $13 \%$ laxatives. Interestingly, among those over 18 years, no one had constipation. Compared to previous studies these numbers are within the range usually reported after endorectal pull-through [11].
When TERPT was introduced, it rapidly gained wide popularity because TERPT does not require any transabdominal or laparoscopic dissection, minimal postoperative pain enables fast recovery, and TERPT leaves no visible scars. Initially, there were concerns about possible damage to the anal sphincters since the bowel is mobilized solely through the anus and therefore the dilatation of the anal sphincters occurs for a longer period than if most of the colon and rectum is mobilized via laparotomy or laparoscopy. Similar manipulation of the anal canal has been shown to be associated with significant problems in adult patients [15]. One of the first studies that aimed at examining possible damage to the anal sphincters after TERPT, found no significant difference in manometric findings or stooling patterns in 26 patients 23 and 14 months after LERTP and TERPT respectively [16]. In another study of 42 patients, continence scores were two-fold better in those with a transabdominal procedure than in those that had undergone TERPT [17], and among 39 patients beyond three years of age, there was an obvious trend towards more soiling in the TERPT group although this did not reach statistical significance [18]. Stensrud et al. performed anal endosonography in 50 patients after endorectal pull-through and found that scar tissue in the internal anal sphincter was significantly more common in those operated with TERPT compared to those operated with LERPT [6]. In these patients scarring of the internal anal sphincter was significantly associated with daily incidents of fecal incontinence. Although some reports indicate more incontinence after TERPT as compared to LERPT, there are other studies showing no significant difference between the two operative approaches. A recent metaanalysis including 405 patients from five studies compared functional results after LERPT and TERPT and did not find any statistical significant difference in postoperative bowel function [19]. All the included studies in the meta-analysis were retrospective, there was treatment selection prior to inclusion, and follow-up times were short. Furthermore, lack of statistical power may also have influenced results. Data from some of the patients in this series were recently published, and the operative approach did not seem to influence outcome in the 57 patients who answered the BFS questionnaire [20]. Our finding of inferior result after TERPT is worrying since TERPT probably is the most popular operation to treat rectosigmoid aganglionosis. However, our study was not designed to compare the two different operative approaches, and we strongly encourage that further studies are performed to specifically compare bowel function after LERPT and TERPT. To efficiently do so, a prospective, randomized, multicenter design should be employed.

Several reports have shown that having a syndrome associates with poor functional prognosis. Our data are in line with these findings as fecal accidents and constipation were significantly more frequent among syndromic than nonsyndromic patients. For this reason, some 
authors choose to exclude syndromic patients from their reports of functional outcome [21]. Since functional outcome in syndromic and nonsyndromic patients is so different, this must be taken into consideration when parents are informed about prognosis for their particular child.

Many of those who care for HD patients have an impression that bowel function improves with time [12,22-24]. Older patients not having a stoma or an ACE did better than the younger ones as illustrated by higher BFS scores and better individual parameters of fecal control among the oldest patients. It has previously been shown that minor problems related to fecal staining or constipation are not uncommon in the general population, but that these problems resolve after puberty [10]. For other congenital anorectal conditions it has also been demonstrated that bowel function improves as the patients get older [25]. It is reasonable to presume that patients learn to adapt, contributing to improving fecal control as they get older. Interestingly, contrary to improved BFS scores in the older patients, the percentage of patients reporting socially restrictive bowel problems did not decline with increasing age. This is not surprising since older patients probably are more affected by even minor incontinence problems than younger ones. It is important to take this into consideration in the follow-up of adolescents with anorectal disorders. Lastly, even though most patients experience fewer functional problems at older age, others may not improve with increasing age. In the present series, age at follow-up was not a significant predictor for poor outcome. It is likely that agerelated adaption is insufficient in those with the most serious functional problems owing to a technically failed operation causing permanent sphincter damage and/or loss of anal sensation.

When neonatal repair of HD was introduced, it was a concern that particularly incontinence might be a problem. Several studies have shown good short-term results of neonatal repair [26]. Our results support that primary pull-through in babies is safe and that age at operation is not a predictor of poor outcome at long-term follow-up.

Postoperative HAEC was reported in one third of the patients. The frequency of postoperative HAEC varies considerably in the literature, and the use of different definitions of HAEC is probably an important explanation for this variety $[27,28]$. An interesting finding from our study is that soiling at follow-up was more frequent in those who had experienced postoperative HAEC. Similar findings have been reported from a subgroup of this patient population, and the results from this larger patient population support that there is an increased risk in patients with postoperative HAEC to have problems with fecal incontinence [20]. It has not been reported earlier that those who experience abdominal discomfort from milk, have an increased frequency of postoperative HAEC. We have no apparent explanation for this surprising observation. However, it seems reasonable to inform parents that some form of milk hypersensitivity is common among HD patients, and that those who get HAEC may refrain from cow's milk to test if that affects symptoms.

In addition to the large number of patients, the main strength of this study is that patient-reported functional outcome has been examined by independent staff using an extensively validated questionnaire $[5,8,10]$. To the best of our knowledge, this is the largest study where long-term functional outcome after transanal endorectal pull-through has been investigated according to these principles. The fact that centers with relatively few patients were included, may also be viewed as an advantage. Many centers in both Europe and the US treat less than ten new HD patients per year, and it is important to report results from such institutions as well, not only series where one surgeon has operated a high number of patients. There are, however, some limitations of this study. Despite the low dropout rate of $20 \%$, it remains unknown whether the functional outcome is different in those who responded and those who did not. The operations at the four centers have been performed according to the same principles. Still, there may have been small variations in the surgical technique. Lastly, the number of postoperative HAEC episodes was based on parents' reports as well as medical records, and it is not unlikely that HAEC episodes may have been missed as well as viral infections may have been interpreted as HAEC.

To conclude, this multicenter study of a large cohort of HD patients with rectosigmoid aganglionosis shows that a significant number of patients continue to have bowel problems for many years after definite surgery, and some have significant problems when they reach adulthood. Although most aspects of fecal control significantly improved with increasing patient age, these findings imply that HD patients need long term follow-up extending into adulthood. Further clinical trials are warranted to clarify whether TERPT is associated with inferior functional outcomes.

\section{Acknowledgments}

The authors would like to thank nurse practitioner Astri Austrheim for conducting the telephone interviews with the patients.

\section{References}

[1] Ryan ET, Ecker JL, Christakis NA, et al. Hirschsprung's disease: associated abnormalities and demography. J Pediatr Surg 1992;27:76-81.

[2] Lof Granstrom A, Svenningsson A, Hagel E, et al. Maternal risk factors and perinatal characteristics for Hirschsprung disease. Pediatrics 2016;138:1508-12.

[3] Kenny SE, Tam PK, Garcia-Barcelo M. Hirschsprung's disease. Semin Pediatr Surg 2010;19:194-200.

[4] Bradnock TJ, Walker GM. Evolution in the management of Hirschsprung's disease in the UK and Ireland: a national survey of practice revisited. Ann R Coll Surg Engl 2011;93:34-8.

[5] Jarvi K, Laitakari EM, Koivusalo A, et al. Bowel function and gastrointestinal quality of life among adults operated for Hirschsprung disease during childhood: a population-based study. Ann Surg 2010;252:977-81.

[6] Stensrud KJ, Emblem R, Bjornland K. Anal endosonography and bowel function in patients undergoing different types of endorectal pull-through procedures for Hirschsprung disease. J Pediatr Surg 2015;50:1341-6.

[7] Wester T, Rintala RJ. Early outcome of transanal endorectal pull-through with a short muscle cuff during the neonatal period. J Pediatr Surg 2004;39:157-60.

[8] Rintala RJ, Lindahl HG, Rasanen M. Do children with repaired low anorectal malformations have normal bowel function? J Pediatr Surg 1997;32:823-6.

[9] Rintala RJ, Lindahl H. Is normal bowel function possible after repair of intermediate and high anorectal malformations? J Pediatr Surg 1995;30:491-4.

[10] Kyrklund K, Koivusalo A, Rintala RJ, et al. Evaluation of bowel function and fecal continence in 594 Finnish individuals aged 4 to 26 years. Dis Colon Rectum 2012; 55:671-6.

[11] Neuvonen MI, Kyrklund K, Lindahl HG, et al. A populationbased, complete follow-up of 146 consecutive patients after transanal mucosectomy for Hirschsprung disease. J Pediatr Surg 2015;50:1653-8.

[12] Granstrom AL, Husberg B, Nordenskjold A, et al. Laparoscopic-assisted pullthrough for Hirschsprung's disease, a prospective repeated evaluation of functional outcome. J Pediatr Surg 2013;48:2536-9.

[13] Kim AC, Langer JC, Pastor AC, et al. Endorectal pull-through for Hirschsprung's disease-a multicenter, long-term comparison of results: transanal vs transabdominal approach. J Pediatr Surg 2010;45:1213-20.

[14] Mattioli G, Pini Prato A, Giunta C, et al. Outcome of primary endorectal pull-through for the treatment of classic Hirschsprung disease. J Laparoendosc Adv Surg Tech 2008;18:869-74.

[15] van Tets WF, Kuijpers JH, Tran K, et al. Influence of Parks' anal retractor on anal sphincter pressures. Dis Colon Rectum 1997;40:1042-5.

[16] Van Leeuwen K, Geiger JD, Barnett JL, et al. Stooling and manometric findings after primary pull-throughs in Hirschsprung's disease: perineal versus abdominal approaches. J Pediatr Surg 2002;37:1321-5.

[17] El-Sawaf MI, Drongowski RA, Chamberlain JN, et al. Are the long-term results of the transanal pull-through equal to those of the transabdominal pull-through? A comparison of the 2 approaches for Hirschsprung disease. J Pediatr Surg 2007;42:41-7.

[18] Romero P, Kroiss M, Chmelnik M, et al. Outcome of transanal endorectal vs. transabdominal pull-through in patients with Hirschsprung's disease. Langenbecks Arch Surg 2011;396:1027-33.

[19] Thomson D, Allin B, Long AM, et al. Laparoscopic assistance for primary transanal pull-through in Hirschsprung's disease: a systematic review and meta-analysis. BMJ Open 2015;5(3):e006063.

[20] Neuvonen MI, Kyrklund K, Rintala RJ, et al. Bowel function and quality of life after transanal endorectal pull-through for Hirschsprung disease: controlled outcomes up to adulthood. Ann Surg 2016 [epublication ahead of print].

[21] Miyano G, Koga H, Okawada M, et al. Rectal mucosal dissection commencing directly on the anorectal line versus commencing above the dentate line in laparoscopyassisted transanal pull-through for Hirschsprung's disease: prospective mediumterm follow-up. J Pediatr Surg 2015;50:2041-3.

[22] Rescorla FJ, Morrison AM, Engles D, et al. Hirschsprung's disease. Evaluation of mortality and long-term function in 260 cases. Arch Surg 1992;127(8):934-41.

[23] Conway SJ, Craigie RJ, Cooper LH, et al. Early adult outcome of the Duhamel procedure for left-sided Hirschsprung disease-a prospective serial assessment study. J Pediatr Surg 2007;42:1429-32. 
[24] Aworanti OM, McDowell DT, Martin IM, et al. Does functional outcome improve with time postsurgery for Hirschsprung disease? Eur J Pediatr Surg 2016;26: 192-9.

[25] Rintala RJ, Lindahl HG. Fecal continence in patients having undergone posterior sagittal anorectoplasty procedure for a high anorectal malformation improves as adolescence, as constipation disappears. J Pediatr Surg 2001;36:1218-22.
[26] Pini Prato A, Gentilino V, Giunta C, et al. Hirschsprung disease: do risk factors of poor surgical outcome exist? J Pediatr Surg 2008;43:612-9.

[27] Frykman PK, Short SS. Hirschsprung-associated enterocolitis: prevention and therapy. Semin Pediatr Surg 2012;21:328-35.

[28] Demehri FR, Halaweish IF, Coran AG, et al. Hirschsprung-associated enterocolitis: pathogenesis, treatment and prevention. Pediatr Surg Int 2013;29:873-81. 